\title{
Template games and differential linear logic
}

\author{
Paul-André Melliès \\ CNRS and Université Paris Diderot
}

\begin{abstract}
We extend our template game model of multiplicative additive linear logic (MALL) with an exponential modality of linear logic (LL) derived from the standard categorical construction Sym of the free symmetric monoidal category. We obtain in this way the first game semantics of differential linear logic (DiLL). Its formulation relies on a careful and healthy comparison with the model of distributors and generalised species designed ten years ago by Fiore, Gambino, Hyland and Winskel. Besides the resolution of an old open problem of game semantics, the study reveals an unexpected and promising convergence between linear logic and homotopy theory.
\end{abstract}

\section{INTRODUCTION}

Looking backwards into the history of the field, there is little doubt that the bicategorical interpretation of linear logic based on distributors and generalised species [10] has been a turning point in the mathematical semantics of linear proofs and programs. In this model of linear logic, every formula $A$ is interpreted as a small category $\llbracket A \rrbracket$ and every derivation tree

$$
\begin{gathered}
\pi \\
\vdots \\
\hline A_{1}, \ldots, A_{n} \vdash B
\end{gathered}
$$

is interpreted as a distributor (or profunctor)

$$
\llbracket \pi \rrbracket: \quad \llbracket A_{1} \rrbracket \otimes \ldots \otimes \llbracket A_{n} \rrbracket \longrightarrow \backslash \llbracket B \rrbracket .
$$

This interpretation of linear logic may be understood as a "categorification" of the original relational semantics of the logic, where sets are replaced by categories, and relations by distributors. Recall that a distributor

$$
M: A \longrightarrow B
$$

is defined as a functor

$$
M: A \times B^{o p} \longrightarrow \text { Set }
$$

where Set denotes the category of sets and functions. The bicategory Dist of small categories and distributors is symmetric monoidal with tensor product $A, B \mapsto A \otimes B$ of small categories defined as their usual cartesian product $A, B \mapsto A \times B$ in the category Cat of small categories and functors, and with tensorial unit $\mathbb{1}$ defined as the terminal category. The bicategory Dist is moreover *-autonomous (and in fact compact closed) with linear negation defined as the operation $A \mapsto A^{o p}$ of turning a category $A$ into its opposite category $A^{o p}$. This means that there exists a family of isomorphisms between the categories of distributors

$$
\varphi_{A, B, C}: \operatorname{Dist}(A \otimes B, C) \cong \operatorname{Dist}\left(B, A^{o p} \otimes C\right)
$$

natural in $A, B$ and $C$, which provides a form of linear currification to the bicategory Dist.

\section{A. The exponential modality Sym}

One nice aspect of the categorified semantics is that the exponential modality $A \mapsto ! A$ of linear logic is interpreted using the 2-monad

$$
\text { Sym : Cat } \longrightarrow \text { Cat }
$$

which transports every small category $A$ to the free symmetric monoidal category $\operatorname{Sym} A$ generated by $A$. The category $\operatorname{Sym} A$ has objects defined as the finite sequences (or words) $w=a_{1} \ldots a_{n}$ of objects $a_{1}, \ldots, a_{n}$ of the category $A$, and morphisms

$$
\left(\sigma, f_{1} \ldots f_{n}\right): a_{1} \ldots a_{n} \longrightarrow b_{1} \ldots b_{n}
$$

defined as pairs $\left(\sigma, f_{1} \ldots f_{n}\right)$ consisting of a permutation $\sigma \in S_{n}$ on the set of $n$ elements, together with a sequence (or word) of morphisms of the category $A$

$$
f_{k}: a_{k} \longrightarrow b_{\sigma(k)} \quad \text { for } 1 \leq k \leq n \text {. }
$$

As a strict monoidal category, the category $\mathbf{S y m} A$ comes equipped with a pair of functors

$$
\begin{array}{r}
\otimes_{A}: \operatorname{Sym} A \times \operatorname{Sym} A \longrightarrow \operatorname{Sym} A \\
I_{A}: \mathbb{1} \longrightarrow \operatorname{Sym} A
\end{array}
$$

with the tensor product $\otimes_{A}$ defined as concatenation, and the tensor unit $I_{A}$ defined as the empty word. Equipped with this structure, Sym $A$ defines a monoid in the category Cat, which is only commutative up to a natural isomorphism noted $\gamma$ and called the symmetry of the monoidal category:

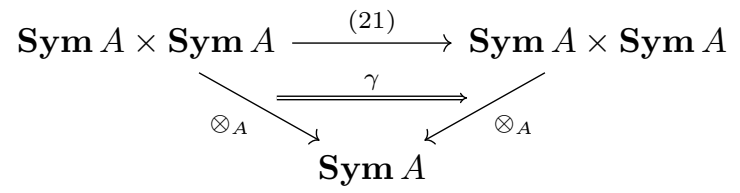

where the functor (21) denotes the symmetry of the cartesian category Cat. 


\section{B. The symmetric monoid and comonoid structures}

The exponential modality $A \mapsto ! A$ of linear logic is interpreted in Dist by turning and extending the 2monad (1) into a 2-comonad

$$
\text { Sym : Dist } \longrightarrow \text { Dist }
$$

using a distributivity law between Sym and the presheaf construction in Cat, see [10] for details. Then, one makes great usage of the following basic observation: every functor between small categories

$$
F \quad: \quad A \longrightarrow B
$$

induces an adjoint pair $L_{F} \dashv R_{F}$ of distributors

$$
L_{F}: A \longrightarrow B \quad R_{F}: B \longrightarrow A
$$

in the bicategory Dist, where the distributors are defined as the functors (or presheaves) below:

$$
\begin{aligned}
& L_{F}(b, a)=B(F b, a): B^{o p} \times A \longrightarrow \text { Set } \\
& R_{F}(a, b)=B(a, F b): A^{o p} \times B \longrightarrow \text { Set }
\end{aligned}
$$

The comonoid structure $\left(\operatorname{Sym} A, d_{A}, e_{A}\right)$ of the exponential modality (4) is then defined by the distributors

$$
\begin{aligned}
& d_{A}=R_{\otimes_{A}} \quad: \quad \operatorname{Sym} A \longrightarrow \operatorname{Sym} A \otimes \operatorname{Sym} A \\
& e_{A}=R_{I_{A}} \quad: \quad \operatorname{Sym} A \longrightarrow \mathbb{1}
\end{aligned}
$$

right adjoints to the functors $\otimes_{A}$ and $I_{A}$ defining the monoidal structure of $\operatorname{Sym} A$ in (2). This reveals an important difference between the bicategorical model in Dist and more traditional categorical models of linear logic: the comonoid ( $\left.\mathbf{S y m} A, d_{A}, e_{A}\right)$ is not commutative as one would expect, but only symmetric in the symmetric monoidal bicategory Dist. This means that Sym $A$ is only commutative up to the isomorphism (5) obtained by transposing the isomorphism (3) from Cat to Dist in the following way:

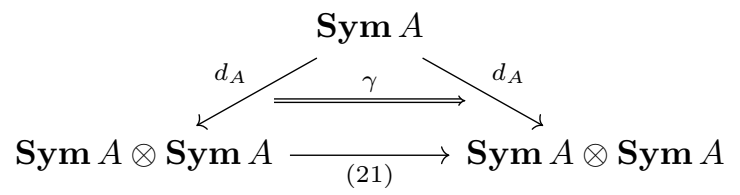

where the distributor (21) denotes in this context the symmetry of the tensor product in Dist.

\section{The Seely equivalence}

The fact that one needs to relax in (5) an equation like commutativity into a natural isomorphism like symmetry is an important and fascinating aspect of the model of distributors. Interestingly, the same bicategorical phenomenon occurs when one considers the well-known Seely isomorphism of linear logic:

$$
!(A \& B) \cong ! A \otimes ! B .
$$

In order to interpret this equation of linear logic as a distributor, one starts by considering the concatenation functor

$$
\text { concat }_{A, B}: \mathbf{S y m} A \times \mathbf{S y m} B \longrightarrow \operatorname{Sym}(A+B)
$$

which takes two sequences $u=a_{1} \cdots a_{p}$ and $v=b_{1} \cdots b_{q}$ of objects of $A$ and of $B$ and concatenates them into the sequence

$$
\operatorname{concat}_{A, B}(u, v)=a_{1} \cdots a_{p} \cdot b_{1} \cdots b_{q}
$$

of objects of $A+B$. The distributor interpreting the Seely isomorphism

$$
\operatorname{Sym}(A \& B) \longrightarrow \operatorname{Sym} A \otimes \operatorname{Sym} B
$$

is then defined as the right adjoint of the concatenation functor, where we write $A \& B=A+B$ for the disjoint union of the categories $A$ and $B$. The important point is that the functor concat $_{A, B}$ is an equivalence of categories (moreover injective on objects), but not an isomorphism of categories. The reason is that an object $w \in \operatorname{Sym}(A+B)$ generally consists of a sequence of objects of $A$ and $B$ shuffled in an arbitrary order, while concat $_{A, B}$ transports a pair $(u, v) \in \mathbf{S y m} A \times \mathbf{S y m} B$ into a sequence (8) where all the objects of $A$ appear before the objects of $B$. From this follows that the distributor (9) is not an isomorphism in the bicategory Dist, but simply an equivalence. In other words, the traditional Seely isomorphism (6) is replaced by a Seely equivalence (9) in the model of distributors.

\section{A model of differential linear logic}

Once the exponential modality of linear logic has been interpreted as $A \mapsto \operatorname{Sym} A$, it appears that the model of distributors does not just provide a mathematical interpretation of linear logic (LL) but also of differential linear logic (DiLL). One main reason is that the exponential modality $\operatorname{Sym} A$ comes equipped with a monoid structure in Dist

$$
\begin{array}{r}
m_{A}=L_{\otimes_{A}}: \quad \operatorname{Sym} A \otimes \operatorname{Sym} A \longrightarrow \operatorname{Sym} A \\
u_{A}=L_{I_{A}}: \mathbb{1} \longrightarrow \operatorname{Sym} A
\end{array}
$$

whose multiplication and unit are the left adjoint distributors associated to the monoid structure of Sym $A$ in Cat mentioned in (2). It is worth stressing the fact that the monoid structure $\left(\operatorname{Sym} A, m_{A}, u_{A}\right)$ and the comonoid structure $\left(\mathbf{S y m} A, d_{A}, e_{A}\right)$ are the left and right adjoint avatars in Dist of the very same monoid structure $\left(\operatorname{Sym} A, \otimes_{A}, I_{A}\right)$ in Cat. As required by a model of DiLL, the monoid and comonoid structure of Sym $A$ define together a bimonoid (also called bialge- 
bra) structure in Dist. Again, this bimonoid structure is only up to an invertible natural transformation:

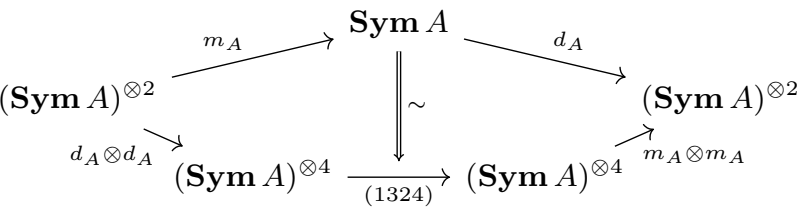

where we write $(\operatorname{Sym} A)^{\otimes n}$ for the $n$-th tensorial power of Sym $A$, and (1324) for the expected distributor in the symmetric monoidal category Dist. One explanation for this structure of bimonoid is the following one: the disjoint sum $A+B$ of two small categories $A$ and $B$ is at the same time their cartesian sum $A \oplus B$ and their cartesian product $A \& B$, in an appropriate bicategorical sense. Every object $A$ thus comes equipped with a monoid and a comonoid structure, with multiplication and comultiplication

$$
\begin{aligned}
\nabla_{A}: A \oplus A \longrightarrow A & \Delta_{A}: A \longrightarrow A \oplus A \\
\nabla_{A}^{0}: \mathbb{D} \longrightarrow A & \Delta_{A}^{0}: A \longrightarrow \mathbb{P}
\end{aligned}
$$

defined as the left and right adjoint distributors associated to the canonical functors $A+A \rightarrow A$ and $\mathbb{D} \rightarrow A$ in Cat, where $\mathbb{D}$ denotes the empty category and initial object of Cat. By the universal nature of their definition, the multiplication $\nabla_{A}$ and the comultiplication $\Delta_{A}$ are equipped with a bimonoid structure in Dist, once again up to an invertible natural transformation:

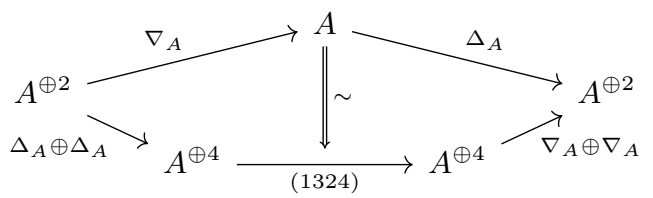

Now, the important point to notice is that the exponential modality $A \mapsto \operatorname{Sym} A$ together with the family of Seely equivalences

$$
\operatorname{Sym}\left(A^{\oplus n}\right) \longrightarrow(\operatorname{Sym} A)^{\otimes n}
$$

defines a (lax and oplax) symmetric monoidal bifunctor

$$
\text { Sym } \quad: \quad(\text { Dist }, \oplus, \mathbb{D}) \longrightarrow(\text { Dist }, \otimes, \mathbb{1})
$$

which transports the "additive" monoid, comonoid and bimonoid structure of $A$ in (Dist, $\oplus, \mathbb{O}$ ) to the "multiplicative" monoid, comonoid and bimonoid structure of Sym $A$ in $($ Dist, $\otimes, \mathbb{1})$. One recognizes here a familiar pattern already in the relational semantics of DiLL.

\section{E. A model of differential linear logic (continued)}

At this stage, one would like to understand how the differential of DiLL is interpreted in the model of distributors. To that purpose, one starts from the families of functors

$$
\eta_{A} \quad: \quad A \longrightarrow \operatorname{Sym} A
$$

defining the unit of the 2-monad $A \mapsto \operatorname{Sym} A$ in Cat. The functor may be post-composed with the functor $\otimes_{A}$ in order to obtain the functor

$$
\text { append }_{A}: A \times \operatorname{Sym} A \longrightarrow \operatorname{Sym} A
$$

which transports a pair $(a, u) \in A \times \operatorname{Sym} A$ to the sequence $a \cdot u \in \operatorname{Sym} A$ where the object $a \in A$ has been appended to the word $u \in \operatorname{Sym} A$. The differential is then interpreted in Dist as the left adjoint distributor

$$
\partial_{A} \quad: \quad A \otimes \operatorname{Sym} A \longrightarrow \operatorname{Sym} A
$$

associated to the functor (11) just defined. The codereliction and dereliction morphisms

$$
\operatorname{coder}_{A}: A \longrightarrow \operatorname{Sym} A \quad \operatorname{der}_{A}: \operatorname{Sym} A \longrightarrow A
$$

are interpreted as the left and right adjoint distributors associated to the functor (10) respectively. The composite of the codereliction with the comultiplication

$$
A \stackrel{\operatorname{coder}_{A}}{\longrightarrow} \operatorname{Sym} A \stackrel{d_{A}}{\longrightarrow} \operatorname{Sym} A \otimes \operatorname{Sym} A
$$

is equal to the disjoint sum of the two distributors

$$
\begin{aligned}
& A \stackrel{\operatorname{coder}_{A}}{\longrightarrow} \operatorname{Sym} A \stackrel{u_{A} \otimes \mathbf{S y m} A}{\longrightarrow} \operatorname{Sym} A \otimes \operatorname{Sym} A \\
& A \stackrel{\operatorname{Sym} A \otimes u_{A}}{\longrightarrow} \operatorname{Sym} A \otimes \operatorname{Sym} A
\end{aligned}
$$

Here, the disjoint sum of two distributors

$$
M, N: A \longrightarrow B
$$

is the distributor $M+N$ defined using the convolution product associated to the "additive" comonoid and monoid structures of $A$ and $B$ :

$$
A \stackrel{\Delta_{A}}{\longrightarrow} A \oplus A \stackrel{M \oplus N}{\longrightarrow} B \oplus B \stackrel{\nabla_{B}}{\longrightarrow} B
$$

or more directly defined as the presheaf

$$
M+N \quad: \quad(b, a) \quad \mapsto \quad M(b, a) \uplus N(b, a)
$$

where $\uplus$ denotes the disjoint union of sets. This property of the codereliction ensures that the Leibniz rule is satisfied, in the technical sense that the composite distributor

$$
A \otimes \operatorname{Sym} A \stackrel{\partial_{A}}{\longrightarrow} \operatorname{Sym} A \stackrel{d_{A}}{\longrightarrow} \operatorname{Sym} A \otimes \operatorname{Sym} A
$$

is isomorphic to the sum of the two distributors obtained by precomposing the distributor

$$
A \otimes \operatorname{Sym} A \stackrel{A \otimes d_{A}}{\longrightarrow} A \otimes \operatorname{Sym} A \otimes \operatorname{Sym} A
$$

with the two (different) distributors represented below:

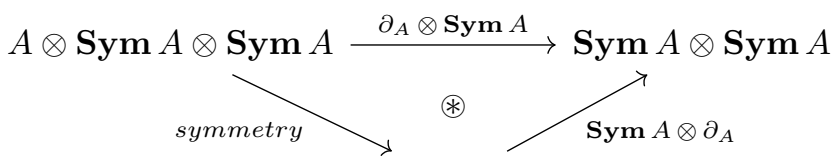

$\operatorname{Sym} A \otimes A \otimes \operatorname{Sym} A$ 


\section{F. What this paper is about}

Our main purpose in the present paper will be to revisit and refine the exponential modality $A \mapsto \operatorname{Sym} A$ of the model of distributors just discussed, in order to adapt it to our recent template game semantics of multiplicative additive linear logic (MALL). The exercise is particularly instructive, since we obtain in this clean and principled way the first game semantics of differential linear logic (DiLL). As we will see, one main difference between the two models (distributors and games) is that template games are based on functorial spans between categories, instead of distributors. Shifting from distributors to functorial spans will reveal a number of fundamental structures hidden in the model of distributors. Surprisingly, these structures are related to the homotopical nature of categories, and more specifically to the canonical Quillen model structure (also called folk model structure) on the category Cat. This model structure is based on the following classification of functors between categories:

weak equivalences: categorical equivalences

fibrations:

cofibrations:

isofibrations

functors injective on objects

A hint of this unexpected convergence between linear logic and homotopy theory lies already in the fact mentioned earlier that the concatenation functor concat $_{A, B}$ defining the Seely equivalence (9) is a categorical equivalence injective on objects, and thus an acyclic cofibration (= weak equivalence and cofibration) in the Quillen model structure on Cat. This basic observation will play an important role in the construction of the model since it indicates that the interpretation of proofs as interactive strategies should be considered up to homotopy of simulations, see \$III for details.

\section{G. Template games}

The notion of template game was recently introduced by the author as a unified framework to construct various $*$-autonomous bicategories $\operatorname{Games}(\star)$ of games, strategies and simulations, see [20]. One main advantage and novelty of the framework is that each bicategory Games $(\star)$ is constructed in a uniform manner, using a synchronization template as parameter. The template is noted with the symbol $t$ and also called anchor for that reason. The purpose of the template $t$ is to express in a simple and concise way the scheduling policy of a specific regime of games and strategies. By way of illustration, three different templates were introduced and studied in the original paper, each of them designed to reflect a particular scheduling policy:

\footnotetext{
$t_{\text {alt }}$ for sequential alternating games,

$t_{\text {conc }}$ for concurrent non-alternating games,

$t_{\text {span }}$ for functorial spans with no scheduling.
}

One guiding principle of template games is that the higher algebraic structure of the bicategory Games $(t)$ mirrors the simpler combinatorial structure of the underlying template $t$. Typically, the construction of the bicategory Games $(t)$ relies on the hypothesis that the template $t$ defines an internal category in a given category $\mathbb{S}$ with finite limits, typically chosen as $\mathbb{S}=$ Cat. In order to shorten and simplify the terminology, we call

$$
\text { S-category, \$-functor, natural \$-transformation }
$$

what is traditionally called internal category, internal functor and internal natural transformation in a given category $\mathbb{S}$ with finite limits. Accordingly, we write

$$
\operatorname{Cat}(\mathbb{S})
$$

for the 2-category of $\$$-categories, or internal categories in $\mathbb{S}$. The fact that the bicategory $\operatorname{Games}(t)$ is *autonomous is derived from the following theorem established in [20] which relates the world of bicategories to the world of synchronization templates:

Theorem [20] The bicategory Games $(t)$ of games, strategies and simulations is $*$-autonomous when the S-category $t$ is span-monoidal $*$-autonomous.

One benefit of using templates instead of working directly on bicategories of games and strategies is that it is much easier to check that an $\$$-category $t$ of interest is span-monoidal *-autonomous than it is to establish that the associated bicategory Games( $t)$ is *-autonomous. This general principle is illustrated in [20] by simple and purely combinatorial proofs that the three synchronization templates

$$
\star=\star_{\text {alt }}, \star_{\text {conc }}, t_{\text {span }}
$$

are span-monoidal $*$-autonomous in the category $\mathbb{S}=$ Cat. From this follows that in each case, the bicategory $\operatorname{Games}(t)$ is $*$-autonomous, and has finite products and coproducts. The bicategory Games $(\star)$ thus defines in each case a specific game semantics (sequential, concurrent, span-relational) of multiplicative additive linear logic (MALL).

\section{H. A game semantics of differential linear logic}

In the present paper, we describe at an axiomatic level what structure should be added to a given synchronization template $t$ in order to extend the associated model of MALL with an interpretation of the exponential modality $A \mapsto ! A$. We are guided in that quest by the notion of span-monoidal structure on a $\$$ category $t$ formulated in [20] as a pair of $\mathbb{S}$-categories $t^{\otimes}$ and $t^{I}$ together with a pair of spans of $\mathbb{S}$-functors

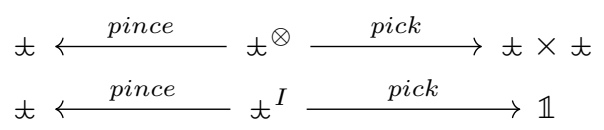


where $\mathbb{1}$ denotes the terminal $\mathbb{S}$-category, and satisfying a number of coherence properties. One asks moreover that the $\mathbb{S}$-functors pick are acute in the sense of [20]. A simple recipe summarised by the sentence

$$
\text { pullback along pick and postcompose with pince }
$$

enables one to derive a bifunctor

$$
\otimes: \operatorname{Games}(t) \times \operatorname{Games}(t) \longrightarrow \operatorname{Games}(t) .
$$

which turns Games(t) into a monoidal bicategory, with unit derived from (13). We would like to extend and adapt this idea in order to interpret the exponential modality in the template game model. To that purpose, we start by observing that the 2-monad Sym preserves pullbacks in the category $\mathbb{S}=$ Cat. From this follows that the image $\operatorname{Sym}(\star)$ of an internal category $t$ is again an internal category. This enables us to define a exponential modality on $t$ as a span of internal functors

$$
t \longleftarrow \text { pince } t^{!} \stackrel{p i c k}{\longrightarrow} \operatorname{Sym}(t)
$$

satisfying a number of coherence properties, see $\$ \sqrt{\mathrm{V}}$ for details. As we will see, one instructive outcome of this work is the unexpected discovery that in order to interpret the exponential modality $A \mapsto ! A$ of linear logic, one should replace the original $*$-autonomous bicategory $\operatorname{Games}(\star)$ by a "homotopy-friendly" bicategory $\operatorname{Games}(\mathbb{F}, t)$ where composition of strategies is defined by homotopy pullbacks instead of usual (and potentially incorrect) categorical pullbacks, see \$III.

\section{Related works}

The idea of connecting linear logic and homotopy theory was explored for the first time by Egger in his $\mathrm{PhD}$ thesis, see [7]. The motivation at the time was to construct a $*$-autonomous category $\mathbb{C}$ equipped with a Quillen model structure $(\mathscr{W}, \mathscr{C}, \mathscr{F})$ where the mix rule $A \otimes B \rightarrow A>8 B$ is a weak equivalence, and to obtain in this way a compact closed category Ho $\mathbb{C}$ as homotopy category. To the author's knowledge, the connection between homotopy theory and the exponential modality $A \mapsto ! A$ of linear logic appears for the first time in the present paper. It provides the latest insight in a long tradition of works devoted to the structure of symmetries between copies in the exponential modality of linear logic, starting from [1] and including [21], [17], [6], [4]. The fact that in the case of $\mathbb{S}=$ Cat, one requires that every strategy $\sigma=\left(S, s, t, \lambda_{\sigma}\right)$ of the model is defined by isofibrations $A \leftarrow S \rightarrow B$ means that every "symmetry" appearing in the games $A$ and $B$ lifts to the support $S$ of the strategy. This homotopy-theoretic assumption is thus reminiscent of the idea advocated in [2], [4] that one should only consider the strategies which are saturated modulo the action of the symmetric group $\Sigma_{n}$ on the tensorial powers $A^{\otimes n}$ of $n$ copies of the game $A$. As additional precursor to this work, let us mention the game semantics of the differential $\lambda$-calculus, the intuitionistic fragment of DiLL, designed in [16] and based on the nondeterministic pointer game semantics formulated by Harmer and McCusker [13].

\section{J. Synopsis of the paper}

After this long and detailed introduction, we recall in $\$$ II how the $*$-autonomous bicategory $\operatorname{Games}(t)$ is constructed in [20]. We then explain in \$III] why homotopy theory plays a central role in our interpretation of the exponential modality from distributors to template games. This leads us to an axiomatic description in \$IV of the basic assumptions on the homotopy structure of the underlying category $\mathbb{S}$ and of the 2-monad Sym. A general construction of the exponential modality is described in $\$ \nabla$. The fact that it defines a model of differential linear logic is established in $8 \mathrm{VI}$. We illustrate the construction by defining in $\$$ VII an exponential modality for the template $t_{\text {alt }}$ of alternating games and strategies. We then conclude in \$VIII.

\section{The BICATEGory of GAMES AND STRATEGIES}

\section{A. Internal categories}

We suppose given a category $\mathbb{S}$ with finite limits, whose objects we find convenient to call spaces. An internal graph $\star$ in such a category $\mathbb{S}$ with finite limits is defined as a pair of spaces (= objects in $\mathbb{S}$ )

$$
\star[0] \quad t[1]
$$

called the space $\star[0]$ of objects and the space $\star[1]$ of maps, together with a pair of morphisms

$$
\star[0] \longleftarrow s
$$

called the source and target morphisms. Typically, an internal graph $t$ in the category $\mathbb{S}=$ Set of sets and functions is just the same thing as a graph. Every internal graph $t$ comes equipped with the space $t[2]$ of composable maps defined as the pullback

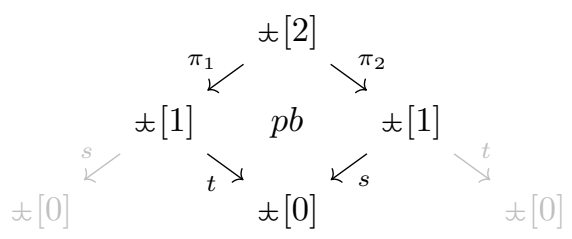

computed in the category $\mathbb{S}$ of spaces. An internal category $t$ is defined as an internal graph equipped with two morphisms

$$
\star[2] \stackrel{m}{\longrightarrow} \star[1] \quad \star[0] \stackrel{e}{\longrightarrow} \star[1]
$$

called composition and identity respectively, and satisfying a number of coherence properties expressing the fact that composition is associative and that identity 
maps are neutral elements. Note that an internal category $t$ in the category $\mathbb{S}=$ Set is just the same thing as a small category.

\section{$B$. The bicategory of games and strategies}

Given an internal category $t$ in the category $\mathbb{S}$ with finite limits, the bicategory Games $(\star)$ of games, strategies and simulations is defined in the following way. Its objects are the pairs $\left(A, \lambda_{A}\right)$ consisting of an object $A$ of the category $\mathbb{S}$ together with a map

$$
\lambda_{A}: A \longrightarrow \star[0]
$$

Its maps (called strategies)

$$
\sigma=\left(S, s, t, \lambda_{\sigma}\right):\left(A, \lambda_{A}\right) \longrightarrow\left(B, \lambda_{B}\right)
$$

are defined as the spans

$$
A \longleftarrow s
$$

with support $S$, together with a map $\lambda_{\sigma}: S \rightarrow \star[1]$ making the diagram below commute:

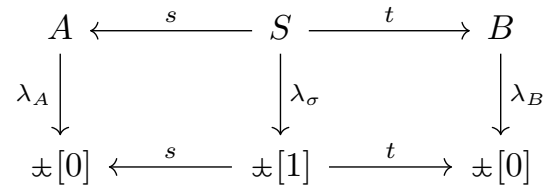

The 2-cells of the bicategory $\operatorname{Games}(\star)$ are the simulations

$$
\theta: \sigma \Longrightarrow \tau: A \longrightarrow B
$$

defined as maps $\theta: S \rightarrow T$ making the diagram below commute:

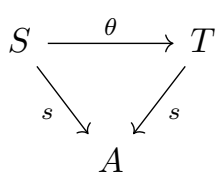

A
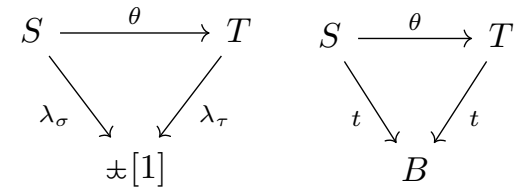

where $S$ is the support of $\sigma$ and $T$ is the support of $\tau$. Two maps (or strategies)

$$
\begin{aligned}
& \sigma=\left(S, s_{S}, t_{S}, \lambda_{\sigma}\right): A \longrightarrow B \\
& \tau=\left(T, s_{T}, t_{T}, \lambda_{\tau}\right): B \longrightarrow C
\end{aligned}
$$

of $\operatorname{Games}(\star)$ are composed in the following way:

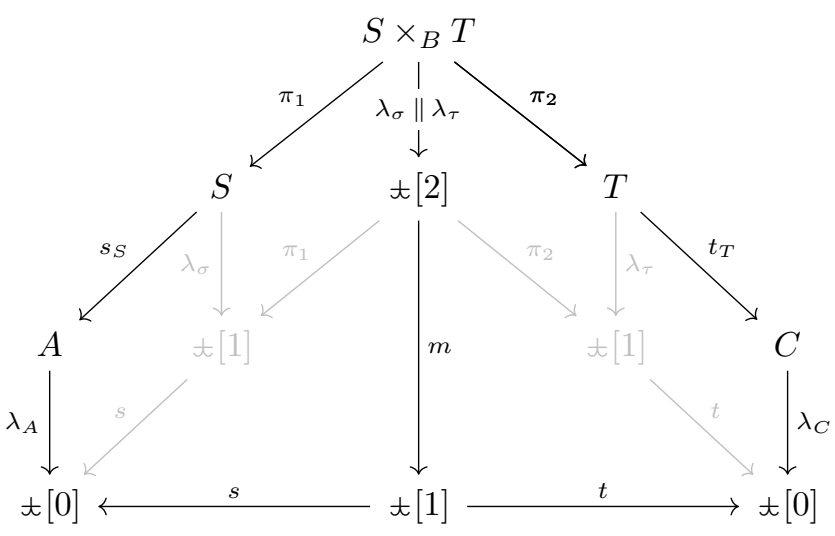

Here, $\lambda_{\sigma} \| \lambda_{\tau}$ denotes the map of $\mathbb{S}$ induced by the pullback diagram (17) and uniquely determined by the equations

$$
\lambda_{\sigma} \circ \pi_{1}=\pi_{1} \circ\left(\lambda_{\sigma} \| \lambda_{\tau}\right) \quad \lambda_{\tau} \circ \pi_{2}=\pi_{2} \circ\left(\lambda_{\sigma} \| \lambda_{\tau}\right)
$$

The identity map

$$
\mathbf{i d}_{A}=\left(A, i d_{A}, i d_{A}, e \circ \lambda_{A}\right): A \longrightarrow A
$$

is constructed in the following way:

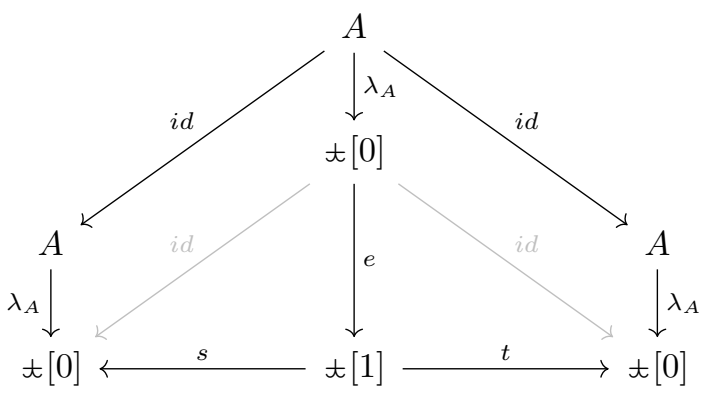

III. THE EMERGENCE OF HOMOTOPY

In this section, we explain why homotopy theory plays a central and necessary role in order to interpret the exponential modality $A \mapsto ! A$ of linear logic in our template game models. To that purpose, we find convenient to work in the special case where $\mathbb{S}=$ Cat is the category of small categories. We start by observing in \$III-A that the hom-category Games $(t)(A, B)$ is in fact a 2-category when the underlying category $\mathbb{S}$ is a 2-category, as it is the case for $\mathbb{S}=$ Cat. Moreover, in the special case when $\mathbb{S}=$ Cat, every isomorphism (26) of functors is interpreted as a cospan (27) of weak equivalences between strategies in $\operatorname{Games}(t)(A, B)$. In order for composition to preserve these weak equivalences, we explain in \$III-B and \$III-C that one needs to compose strategies by homotopy pullbacks instead of usual pullbacks. Finally, we give a brief description in \$III-D of the main contribution of the paper, which is to provide an axiomatic homotopy framework for template games.

\section{A. A troublesome and remarkable phenomenon}

As explained in the introduction (\$I-C), one fundamental benefit of shifting to a 2-categorical model of linear logic like the model of distributors is that the Seely isomorphism (6) is replaced there by a more informative and precise Seely equivalence (9). In the case of Dist, this equivalence (9) is provided by the right adjoint distributor associated to an equivalence (7) of categories living in Cat. The situation becomes even more interesting and subtle when one shifts from Dist to the bicategory Games $(t)$ of games, strategies and simulations associated to a $\mathbb{S}$-category $t$, and more specifically when $\mathbb{S}$ is a 2-category and not just a category. In this situation, the category $\operatorname{Games}(t)(A, B)$ 
of strategies and simulations between two games $A$ and $B$ becomes a 2-category, with 2-cells

$$
\alpha: \theta_{1} \Longrightarrow \theta_{2}: \sigma \Longrightarrow \tau: A \longrightarrow B
$$

between simulations of the form (21) defined as 2-cells

$$
\alpha: \theta_{1} \longrightarrow \theta_{2}: S \longrightarrow T
$$

of the underlying 2-category $\mathbb{S}$. In the important case $\mathbb{S}=$ Cat, we are confronted to this unexpected and troublesome fact that the 2-cells (22) of Games $(\star)(A, B)$ are not preserved by pullbacks of spans in the 2-category $\mathbb{S}=$ Cat. In particular, we cannot expect to turn the bicategory Games( $(t)$ into something like a tricategory of games, strategies and simulations, even when $\mathbb{S}$ is a 2-category like Cat.

\section{$B$. The emergence of cylinder categories}

Luckily, this phenomenon of a purely 2-categorical nature remains invisible in the construction of the *-autonomous bicategory Games( $(t)$ because the construction of Games $(\star)$ relies only on the categorical structure of $\mathbb{S}$, see [20] for details. The phenomenon is likely to reappear however when one decides to equip the bicategory $\operatorname{Games}(t)$ with an exponential modality. In order to explain why, we find convenient to consider the simple case when $t=t_{\text {span }}$ denotes the terminal $\mathbb{S}$-category in $\mathbb{S}=$ Cat. In that case, the bicategory Games $(t)$ coincides with the bicategory $\operatorname{Span}(\mathbb{S})$ of spans in $\mathbb{S}=$ Cat, whose exponential modality is defined as the 2-functor

$$
\text { Sym : } \operatorname{Span}(\mathrm{Cat}) \longrightarrow \operatorname{Span}(\mathrm{Cat})
$$

obtained by lifting the functor Sym in (1), using the fact that it preserves pullbacks in Cat. In order to turn the 2-functor (23) just formulated into an exponential modality, we proceed by analogy with Dist, and observe that every functor $F: A \rightarrow B$ between small categories induces an adjoint pair $L_{F} \dashv R_{F}$ of spans

$$
L_{F}: A \longrightarrow B \quad R_{F}: B \longrightarrow A
$$

in the bicategory $\operatorname{Span}(\mathbf{C a t})$. The two spans $L_{F}$ and $R_{F}$ are respectively defined as:

$$
A \stackrel{i d_{A}}{\longleftarrow} A \stackrel{F}{\longrightarrow} B \quad B \stackrel{F}{\longleftarrow} A \stackrel{i d_{A}}{\longrightarrow} A .
$$

As in the case of the model of distributors, this family of adjoint pairs $L_{F} \dashv R_{F}$ enables us to equip every category of the form Sym $A$ with a comonoid structure

$$
\begin{aligned}
d_{A}=R_{\otimes_{A}} & : \operatorname{Sym} A \longrightarrow \operatorname{Sym} A \otimes \operatorname{Sym} A \\
e_{A}=R_{I_{A}} & : \operatorname{Sym} A \longrightarrow \mathbb{1}
\end{aligned}
$$

as well as with a monoid structure

$$
\begin{array}{r}
m_{A}=L_{\otimes_{A}}: \operatorname{Sym} A \otimes \operatorname{Sym} A \longrightarrow \operatorname{Sym} A \\
u_{A}=L_{I_{A}}: \mathbb{1} \longrightarrow \operatorname{Sym} A
\end{array}
$$

Here, the tensor product $A \otimes B$ of two small categories $A$ and $B$ is defined as their usual (cartesian) product $A \times B$, in the same way as in Dist. At this stage, one would like to carry on the analogy with Dist and lift the symmetry (3) of the monoidal category $\operatorname{Sym} A$ in the same way as it was lifted in $\$$ I-B to the symmetry (5) in the bicategory Dist. However, and this is the whole beauty and novelty of the situation, it turns out that a natural isomorphism $\varphi: F \Rightarrow G$ between functors in Cat

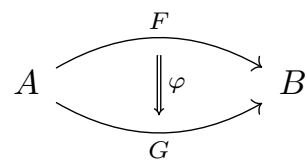

is not transported to a pair of reversible 2-cells

$$
L_{\varphi}: L_{F} \Longrightarrow L_{G} \quad R_{\varphi}: R_{G} \Longrightarrow R_{F}
$$

as it is the case for distributors. Instead, the natural isomorphism $\varphi: F \Rightarrow G$ is transported to a pair of cospans of 2-cells (or simulations) in $\operatorname{Span}(\mathrm{Cat})$

$$
L_{F} \Longleftarrow \widetilde{L}_{\varphi} \Longrightarrow L_{G} \quad R_{F} \Longleftarrow \widetilde{R}_{\varphi} \Longrightarrow R_{G}
$$

defined as follows
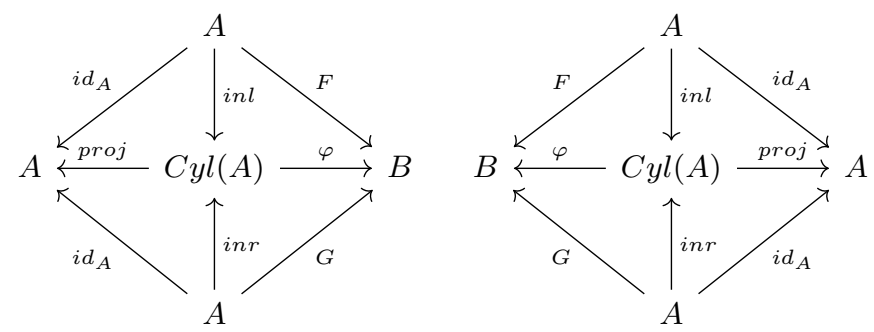

Here, $C y l(A)$ denotes the cylinder category defined as

$$
\operatorname{Cyl}(A)=\sqrt{ } \times A
$$

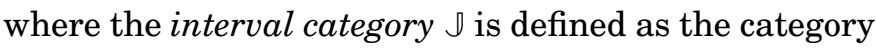
with two objects 0 and 1 and a unique isomorphism $0 \rightarrow 1$ between them. Note that the interval category $\mathbb{J}$ comes equipped with three functors

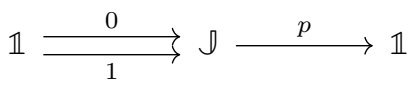

The three functors $i n l$, inr and proj are deduced from that structure on the interval category $\downarrow$ in the following way:

$$
i n l=0 \times A \quad i n r=1 \times A \quad \text { proj }=p \times A .
$$

The spans $\widetilde{L}_{\varphi}$ and $\widetilde{R}_{\varphi}$ are respectively defined as

$$
A \stackrel{\text { proj }}{\longleftarrow} \operatorname{Cyl}(A) \stackrel{\varphi}{\longrightarrow} B \quad B \stackrel{\varphi}{\longleftarrow} \operatorname{Cyl}(A) \stackrel{\text { proj }}{\longrightarrow} A
$$

where the functor $\varphi: C y l(A) \rightarrow B$ internalizes the natural isomorphism (also noted $\varphi$ ) between the functors $F, G: A \rightarrow B$ and thus satisfies the two equations:

$$
F=\varphi \circ i n l \quad G=\varphi \circ i n r
$$

required for $i n l$ and $i n r$ to define simulations in (27). 


\section{A notion of weak equivalence}

What is remarkable here is that the model of functorial spans (and more generally of template games) reveals an unexpected connection between linear logic and homotopy theory, which remained invisible in the original model of distributors. Indeed, the reversible nature of the natural transformation $\varphi: F \Rightarrow G$ in (26) is reflected in the model of functorial spans by the fact that the two functors

$$
\text { inl,inr : } A \Longrightarrow C y l(A)
$$

are equivalences of categories. In order to stress the connection to homotopy theory, we find useful to call weak equivalence any simulation of the form (21) whose underlying morphism $\theta: S \rightarrow T$ is a categorical equivalence in $\mathbb{S}=$ Cat. Note that the definition works for every internal category $t$ in $\mathbb{S}=$ Cat. So, given two template games $A$ and $B$, we write $W_{A, B}$ for the class of weak equivalences in the category $\operatorname{Games}(t)(A, B)$. Using that terminology, the fact that $F$ and $G$ are isomorphic functors in Cat is reflected in our template game semantics by the fact that the cospans (27) of simulations are made of weak equivalences (indicated by the symbol $\sim$ in diagrams)

$$
L_{F} \stackrel{\sim}{\longleftarrow} \widetilde{L}_{\varphi} \stackrel{\sim}{\rightleftharpoons} L_{G} \quad R_{F} \stackrel{\sim}{\longleftarrow} \widetilde{R}_{\varphi} \stackrel{\sim}{\rightleftharpoons} R_{G}
$$

living either inside the category $\operatorname{Games}(t)(A, B)$ or inside the category $\operatorname{Games}(t)(B, A)$. The ongoing discussion convinces us to replace the original category

$$
\operatorname{Games}(\star)(A, B)
$$

of strategies and simulations between two template games $A$ and $B$ by the homotopy category

$$
\operatorname{HoGames}(\star)(A, B)=\operatorname{Games}(\star)(A, B)\left[\mathscr{W}_{A, B}\right]
$$

obtained by localizing the category $\operatorname{Games}(\star)(A, B)$ at the weak equivalences, in other words, by formally inverting the maps (simulations) in $\mathscr{W}_{A, B}$. We will see very soon that, thanks to this localization of the homcategories $\operatorname{Games}(t)(A, B)$ of strategies and simulations, we can lift the symmetry (3) into a symmetry of $\operatorname{Span}(\mathrm{Cat})$ in the just same way as we previously did in the bicategory Dist.

\section{A game model of linear logic up to homotopy}

One main contribution and technical achievement of the paper is to construct for any good synchronization template $t$ a $*$-autonomous bicategory HoGames $(t)$ together with an exponential modality

$$
\text { ! : HoGames }(t) \longrightarrow \operatorname{HoGames}(t)
$$

based on these axiomatic ideas coming homotopy theory. To that purpose, we will make the assumption that our original category $\mathbb{S}$ with finite limits is equipped with a Quillen model structure $(\mathscr{W}, \mathscr{C}, \mathscr{F})$ describing the weak equivalences, cofibrations and fibrations of the category $\mathbb{S}$. Typically, in the case of $\mathbb{S}=$ Cat, we consider the canonical model defined as follows:

$$
\begin{array}{ll}
W & \text { categorical equivalences } \\
\mathscr{C} & \text { functors injective on objects } \\
\mathscr{F} & \text { isofibrations }
\end{array}
$$

From this, one deduces a Quillen model structure $\left(\mathscr{W}_{A, B}, \mathscr{C}_{A, B}, \mathscr{F}_{A, B}\right)$ on the category $\operatorname{Games}(\star)(A, B)$ of strategies and simulations between two games $A$ and $B$, where every simulation $\theta: S \rightarrow T$ of the form (21) inherits its classification

$$
\begin{array}{ll}
\mathscr{W}_{A, B} & \text { weak equivalences in } \operatorname{Games}(t)(A, B) \\
\mathscr{C}_{A, B} & \text { cofibrations in Games }(t)(A, B) \\
\mathscr{F}_{A, B} & \text { fibrations in Games }(t)(A, B)
\end{array}
$$

from the classification of the morphism $\theta: S \rightarrow T$ in the category $\mathbb{S}$. In order to define composition in the bicategory HoGames $(\star)$ with hom-categories

$$
\operatorname{HoGames}(\star)(A, B)=\operatorname{Games}(\star)(A, B)\left[\mathscr{W}_{A, B}\right]
$$

localized at the weak equivalences of $\mathcal{W}_{A, B}$, one needs to replace usual categorical pullbacks by homotopy pullbacks computed in the Quillen model structure $\mathbb{S}$. In order to make our life simpler and avoid unnecessary complications, we will make the assumption that the Quillen model structure $(\mathscr{W}, \mathscr{C}, \mathscr{F})$ is right proper. The assumption tells that the pullback of a weak equivalence $w \in \mathscr{W}$ along a fibration $f \in \mathscr{F}$ is a weak equivalence $w^{\prime} \in \mathscr{W}$. This assumption ensures more generally that every (usual categorical) pullback along a fibration $f \in \mathscr{F}$ computes in fact a homotopy pullback in the model category $\mathbb{S}$. This is in particular the case of the category Cat equipped with its canonical model structure $(\mathscr{W}, \mathscr{C}, \mathscr{F})$.

\section{BASIC ASSUMPTIONS ON $\mathbb{S}$}

In this section, we assume that the category $\mathbb{S}$ comes equipped with a monad Sym and describe what axiomatic properties they should both satisfy. We start by describing in $\$$ IV-A the homotopy structure required of $\mathbb{S}$ and $t$, and then explicate in $\$ \overline{I V-B}$ how the monad Sym should behave and interact with it.

\section{A. A Quillen model structure}

We suppose that the category $\mathbb{S}$ is equipped with a right proper Quillen model structure $(\mathscr{W}, \mathscr{C}, \mathscr{F})$. We suppose that the $\mathbb{S}$-category $\star$ satisfies the following homotopy properties:

Property A. The two spaces $t[0]$ and $t[1]$ in $[15$ of the internal category $t$ are fibrant objects in $\mathbb{S}$, and its structural $\mathbb{S}$-morphisms $s, t, m, e$ in (16) and (18) are fibrations in $\mathbb{S}$. 
Recall that an object $A$ is fibrant in the category $\mathbb{S}$ when the canonical morphism $A \rightarrow \mathbb{1}$ to the terminal object is a fibration. At this stage, we find convenient to define $\mathbb{E}$ as the subcategory of $\mathbb{S}$ consisting of fibrant objects and fibrations $A \rightarrow B$ between them. This enables us to define

\section{$\operatorname{Games}(\mathbb{F}, \star)$}

as the sub-bicategory of Games $(\star)$ consisting of

- the games $\left(A, \lambda_{A}\right)$ whose morphism $\lambda_{A}: A \rightarrow \star[0]$ is a fibration in the category $\mathbb{S}$,

- the strategies $\sigma=\left(S, s, t, \lambda_{\sigma}\right)$ whose morphisms $s$, $t, \lambda_{\sigma}$ are fibrations in the category $\mathbb{S}$,

- the simulations $\theta: \sigma \Rightarrow \tau$ are the same as in $\operatorname{Games}(t)$.

Given two games $A$ and $B$, we write $\mathscr{W}_{A, B}^{\mathbb{F}}$ for the class of weak equivalences between strategies in $\operatorname{Games}(\mathbb{F}, \star)(A, B)$. It appears that the category

$$
\operatorname{HoGames}(\mathbb{F}, \star)(A, B)=\operatorname{Games}(\mathbb{F}, \star)(A, B)\left[\mathscr{W}_{A, B}^{\mathbb{F}}\right]
$$

obtained by inverting the weak equivalences of $\operatorname{Games}(\mathbb{F}, t)(A, B)$ is equivalent to the category HoGames $(\star)(A, B)$ defined in (28). Moreover, by our assumption that the underlying model structure on $\mathbb{S}$ is right proper, the pullbacks of spans in $\operatorname{Games}(\mathbb{F}, t)$ are pullbacks along fibrations, and thus homotopy pullbacks. In particular, composition of strategies in the bicategory $\operatorname{Games}(\mathbb{F}, \star)$ preserves weak equivalences between them. As such, the construction provides an answer and solution to the observations made in \$III Accordingly, we ask that

Property B. All the objects and morphisms defining the $*$-autonomous span-monoidal structure of $t$ are fibrant objects and fibrations in $\mathbb{S}$.

We make a last assumption on the category $\mathbb{S}$, which ensures that the bicategory $\operatorname{Games}(\mathbb{F}, t)$ has finite products provided by the finite sums $(+, \mathbb{O})$ of the underlying category $\mathbb{S}$.

Property C. The finite sum of fibrant objects is fibrant, and for every fibration $f: S \rightarrow A_{1}+A_{2}$ where $S, A_{1}$ and $A_{2}$ are fibrant objects, there exists a unique pair of fibrant objects $S_{1}$ and $S_{2}$ and a unique pair of fibrations $f_{1}: S_{1} \rightarrow A_{1}$ and $f_{2}: S_{2} \rightarrow A_{2}$ up to isomorphism such that the sum $S_{1}+S_{2}$ is isomorphic to $S$ and the fibration $f: S \rightarrow A_{1}+A_{2}$ is induced by universality property from the fibrations $f_{1}$ and $f_{2}$. Similarly, every fibration $f: S \rightarrow \mathbb{O}$ is an isomorphism.

\section{B. The monad Sym and its properties}

Besides the homotopy structure on $\mathbb{S}$, we ask that the category $\mathbb{S}$ comes equipped with a monad

$$
(\mathbf{S y m}, \eta, \mu) \quad: \quad \mathbb{S} \longrightarrow \mathbb{S}
$$

One requires that
Property D. The monad Sym is cartesian.

This means that the functor Sym preserves pullbacks, and that the unit $\eta$ and multiplication $\mu$ of the monad define pullbacks:
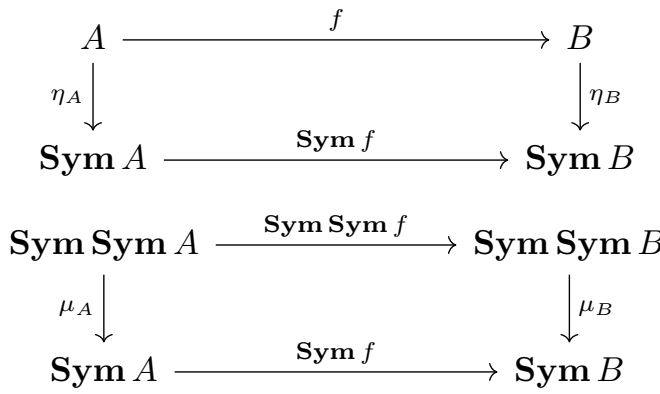

for every morphism $f: A \rightarrow B$ in $\mathbb{S}$. From Property D. we derive the fact that Sym lifts to a functor

$$
\text { Sym : } \operatorname{Cat}(\mathbb{S}) \longrightarrow \operatorname{Cat}(\mathbb{S})
$$

One also requires that

Property E. The monad Sym transports fibrations to fibrations and fibrant objects to fibrant objects. Moreover, every morphism $\eta_{A}$ and $\mu_{A}$ is a fibration.

Note that Property E. implies that the monad Sym restricts to a monad

$$
(\mathbf{S y m}, \eta, \mu) \quad: \quad \mathbb{F} \longrightarrow \mathbb{F}
$$

on the subcategory $\mathbb{F}$ of fibrant objects and fibrations.

Finally, we require as last assumption on Sym that there exists a family of weak equivalences interpreting the Seely isomorphism in the category $\mathbb{S}$. Note that category $\mathbb{S}$ has finite sums $(+, \mathbb{O})$ as a category equipped with a Quillen model structure.

Property F. There exists a family of weak equivalences

$$
\operatorname{Sym} A \times \operatorname{Sym} B \stackrel{\sim}{\longrightarrow} \operatorname{Sym}(A+B) \quad \mathbb{1} \stackrel{\sim}{\rightarrow} \operatorname{Sym} 0
$$

making the expected coherence diagrams of a lax symmetric monoidal structure commute up to left homotopy $\sim_{l}$ in the Quillen model structure. See [15] for a definition of left homotopy, and its relationship to the notion of cylinder object already encountered in \$III-B.

\section{THE EXPONENTIAL MODALITY}

Here, we fix a right proper Quillen category $\mathbb{S}$ together with a monad Sym and a symmetric spanmonoidal S-category $t$ satisfying the Properties A-F formulated in $\$ \mathrm{IV}$. The main purpose of the section is to introduce the notion of exponential modality on the synchronization template $t$. We have seen that the construction of the exponential modality Sym on the bicategory of distributors relies on the fact that every functor $F: A \rightarrow B$ induces a pair $L_{F} \dashv R_{F}$ of adjoint distributors. We proceed similarly here, and establish a similar property for strategies in $\operatorname{Games}(\mathbb{F}, t)$. 


\section{A. Adjunctions}

Suppose given two games $\left(A, \lambda_{A}\right)$ and $\left(B, \lambda_{B}\right)$ whose morphisms $\lambda_{A}$ and $\lambda_{B}$ are fibrations in the category $\mathbb{S}$, and thus objects in the slice category $\mathbb{F} / t[0]$. In that case, every morphism (and thus fibration) $f:\left(A, \lambda_{A}\right) \rightarrow$ $\left(B, \lambda_{B}\right)$ of the category $\mathbb{F} / t[0]$ induces an adjoint pair of strategies

$$
\begin{aligned}
& L_{f}:\left(A, \lambda_{A}\right) \longrightarrow\left(B, \lambda_{B}\right) \\
& R_{f}:\left(B, \lambda_{B}\right) \longrightarrow\left(A, \lambda_{A}\right)
\end{aligned}
$$

in the bicategory $\operatorname{Games}(\mathbb{F}, t)$. The strategies are respectively defined as the morphisms of spans

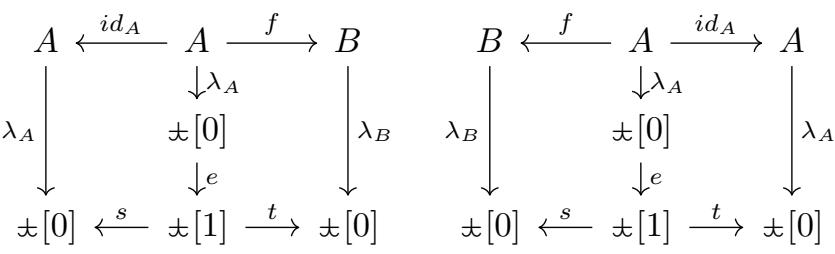

\section{B. The exponential modality (functorial part)}

We are now ready to formulate the functorial part of our definition of exponential modality.

Definition 1 (exponential premodality): An exponential premodality is defined as a \$-category $t !$ together with a span of $\mathbb{S}$-functors

$$
\star \longleftarrow \text { pince } \star^{!} \stackrel{\text { pick }}{\longrightarrow} \operatorname{Sym}(t)
$$

in $\operatorname{Cat}(\mathbb{S})$, where the $\mathbb{S}$-functors pick and pince are acute in the sense of [20]. One requires moreover that the objects and morphisms defining the $\mathbb{S}$-category $t$ ! and the $\mathbb{S}$-functors pick and pince are fibrant objects and fibrations in the category $\mathbb{S}$.

From these assumptions, one obtains a functor

$$
!_{[0]} \quad: \quad \mathbb{S} / \star[0] \longrightarrow \mathbb{S} / \star[0]
$$

defined by transporting every morphism $\lambda_{A}: A \rightarrow \star[0]$ to the pullback of Sym $A$ along pick $[0]$ postcomposed with pince[0], in the following way:

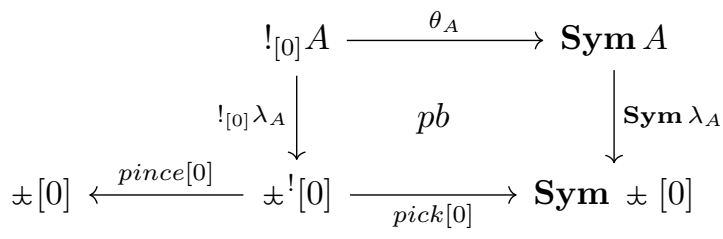

From this definition, it follows that the morphisms $\theta_{A}$ : ![0] $A \rightarrow$ Sym $A$ are fibrations in the category $\mathbb{S}$ and turn the diagram

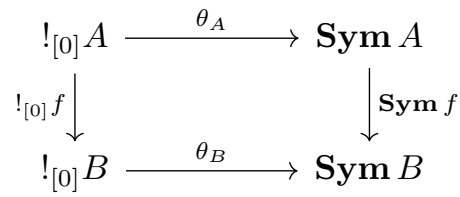

into a pullback diagram in the category $\mathbb{S}$ for every morphism $f: A \rightarrow B$. From this, one deduces easily that the functor (35) transports fibrations into fibrations and fibrant objects into fibrant objects ; and that the functor (35) is moreover cartesian, in the sense that it transports every pullback diagram of $\mathbb{S} / \star[0]$ into a pullback diagram. The span (13) of $\mathbb{S}$-functors induces by the same procedure applied to pick[1] and pince[1] a functor

$$
!_{[1]}: \mathbb{S} / \star[1] \longrightarrow \mathbb{S} / \star[1]
$$

Note that the two functors ! $!_{[0]}$ and ! ${ }_{[1]}$ are related by a pair of natural transformations

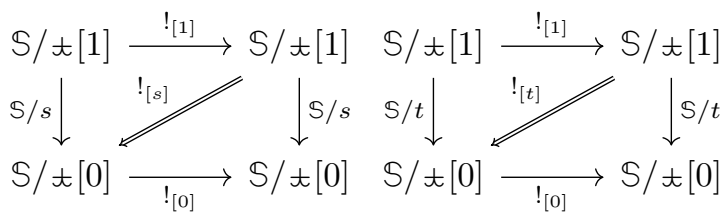

where the functors $\mathbb{S} / s$ and $\mathbb{S} / t$ are the expected functors defined by postcomposition with $s, t: \star[1] \rightarrow \star[0]$. From these structures, one deduces a functor

$$
\text { ! : } \operatorname{Cat}(\mathbb{S}) / t \longrightarrow \operatorname{Cat}(\mathbb{S}) / t
$$

which transports every $\mathbb{S}$-category A equipped with a S-functor $F: \mathbf{A} \rightarrow \star$ to the internal $\$$-category

$$
(! \mathbf{A})[0]=!_{[0]}(\mathbf{A}[0]) \quad(! \mathbf{A})[1]=!_{[1]}(\mathbf{A}[1])
$$

equipped with a $\mathbb{S}$-functor $F^{\dagger}: ! \mathbf{A} \rightarrow \star$ derived from the $\mathbb{S}$-functor $F$. As a form of categorical bootstrap, one obtains in that way the equation $t^{!}=! \star$ where $\star$ comes equipped as an object of $\operatorname{Cat}(\mathbb{S}, t)$ with the identity $\mathbb{S}$-functor $i d: \star \rightarrow \star$ while its image ! $t$ comes equipped with the $\mathbb{S}$-functor pince $: t^{!} \rightarrow t$.

\section{The exponential modality (monadic part)}

At this point, we want to turn the functor (35) into a monad on the category $\mathbb{E} / \star[0]$. To that purpose, we extend the previous definition (Def. 1) of exponential premodality in the following way:

Definition 2 (exponential modality): An exponential modality is an exponential premodality equipped with two $\mathbb{S}$-functors unit : $t \rightarrow$ ! $\rightarrow$ and mult $: ! !_{\star} \rightarrow !_{\star}$ whose components are fibrations of the category $\mathbb{S}$, and defining the pullback diagrams below:

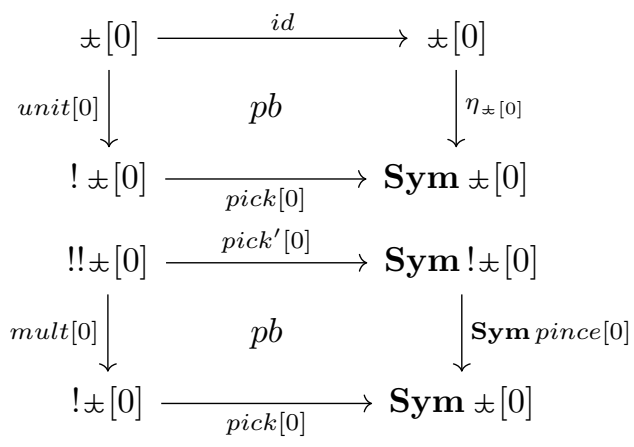


From this additional structure, one deduces that ! $[0]$ defines a monad

$$
!_{[0]} \quad: \mathbb{F} / \star[0] \longrightarrow \mathbb{F} / \star[0]
$$

whose unit and multiplication morphisms

$$
\begin{aligned}
\eta_{A} & : \quad\left(A, \lambda_{A}\right) \longrightarrow !_{[0]}\left(A, \lambda_{A}\right) \\
\mu_{A}: \quad !_{[0]} !_{[0]}\left(A, \lambda_{A}\right) \longrightarrow & \longrightarrow !_{[0]}\left(A, \lambda_{A}\right)
\end{aligned}
$$

are moreover fibrations between fibrant objects in the category $\mathbb{S} / \star[0]$. The counit and comultiplication of the exponential modality

$$
\begin{array}{ll}
\varepsilon_{A} & : \quad !_{[0]}\left(A, \lambda_{A}\right) \longrightarrow \\
\delta_{A} & : \quad !_{[0]}\left(A, \lambda_{A}\right) \longrightarrow\left(A, \lambda_{A}\right) \\
\longrightarrow & \longrightarrow !_{[0]} ![0] \\
{\left[A, \lambda_{A}\right)}
\end{array}
$$

are then defined in the bicategory $\operatorname{Games}(\mathbb{F}, t)$ as the right adjoint strategies (33) associated to the fibrations $\eta_{A}$ and $\mu_{A}$. In order to ensure that $\varepsilon_{A}$ and $\delta_{A}$ are natural, one requires moreover that the diagram below is a pullback

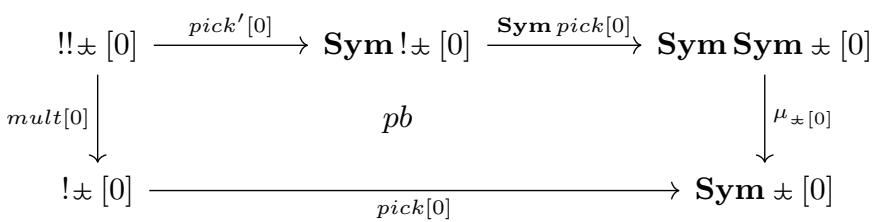

where $p i c k^{\prime}[0]$ is the fibration of $\mathbb{S}$ defined in the pullback diagrams $(40)$. The very same axioms should be required at the degree 1 in order to ensure that the functor (37) defines a monad in $S / \star[1]$ and that it behaves properly.

\section{THE LINEAR-NON-LINEAR ADJUNCTION}

At this point, we are ready to construct the linearnon-linear adjunction between bicategories

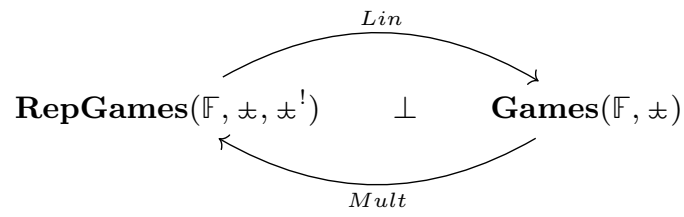

which defines our model of differential linear logic up to homotopy (= localization of the weak equivalences).

\section{A. The Kleisli bicategory}

The bicategory of replicated games

$$
\operatorname{RepGames}\left(\mathbb{F}, \star, \star^{!}\right)
$$

plays the role of Kleisli bicategory in our construction. It has the same objects as the bicategory $\operatorname{Games}(\mathbb{F}, t)$. Then, a map (also called mixed strategy)

$$
\sigma=\left(S, s, t, \lambda_{\sigma}\right) \quad: \quad A \longrightarrow \longrightarrow B
$$

is defined as a span of fibrations

$$
!_{[0]} A \longleftarrow s
$$

together with a fibration

$$
\lambda_{\sigma} \quad: \quad S \longrightarrow \star[1]
$$

making the diagram below commute:

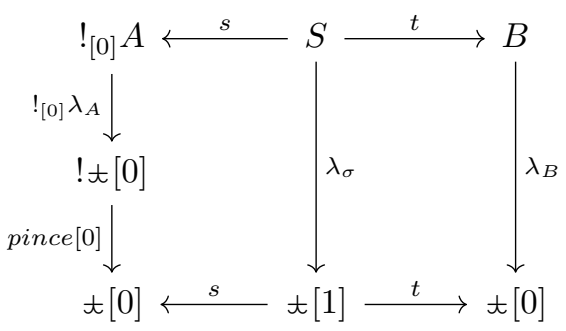

The notion of simulation between mixed strategies is immediate. Composition of mixed strategies is defined using the comonadic structure of the exponential modality. Property C. ensures that the bicategory has finite products noted $(\&, T)$ provided by the finite sums $(+, \mathbb{0})$ of objects in the original category $\mathbb{S}$.

\section{B. The two pseudofunctors Lin and Mult}

The pseudofunctor Lin transports every mixed strategy (42) in the Kleisli bicategory to the strategy

$$
\operatorname{Lin}(\sigma) \quad: \quad !_{[0]} A \longrightarrow !_{[0]} B
$$

with support $!_{[1]} S$ defined as the span of fibrations

$$
!_{[0]} A \stackrel{\mu_{A}}{\longleftarrow} !_{[0]} !_{[0]} A \stackrel{!_{[0]} s 0 !_{[s]}}{\longleftarrow} !_{[1]} S \stackrel{!_{[t]}}{\longrightarrow} !_{[0]} B
$$

together with the fibration

$$
\lambda_{\operatorname{Lin}(\sigma)} \quad: \quad !_{[1]} S \stackrel{!_{[1]} \lambda_{\sigma}}{\longrightarrow} ! \star[1] \stackrel{\text { pince[1] }}{\longrightarrow} \star[1]
$$

Conversely, the pseudofunctor Mult transports every strategy $(19)$ in the bicategory $\operatorname{Games}(\mathbb{F}, \star)$ to the mixed strategy

$$
\operatorname{Mult}(\sigma): A \longrightarrow B
$$

with same support $S$ and defined as the span

$$
!_{[0]} A \longleftarrow \eta_{A}-s \longleftarrow \stackrel{t}{\longleftrightarrow} !_{[0]} B
$$

with same underlying fibration $\lambda_{M u l t(\sigma)}=\lambda_{\sigma}$.

\section{The linear-non-linear adjunction}

The adjunction Lin $\dashv$ Mult between Lin and Mult relies on the fact that the categories are isomorphic

$$
\begin{gathered}
\operatorname{Games}(\mathbb{F}, \star)(\operatorname{Lin}(A), B) \\
\cong \operatorname{RepGames}\left(\mathbb{F}, \star, \star^{!}\right)(A, \operatorname{Mult}(B))
\end{gathered}
$$

for every pair of games $A$ and $B$ whose underlying morphisms $\lambda_{A}: A \rightarrow \star[0]$ and $\lambda_{B}: B \rightarrow \star[0]$ are 
fibrations. Moreover, by Property F. the left adjoint Lin comes equipped with a family of morphisms

$$
\operatorname{Lin} A \otimes \operatorname{Lin} B \longrightarrow \operatorname{Lin}(A \& B) \quad \mathbb{1} \longrightarrow \operatorname{Lin} \top
$$

which interpret the Seely isomorphism, and which become bicategorical equivalences in HoGames(t), after localization of the simulations which are weak equivalences in Games( $(\mathbb{F}, t)$. Note that the pseudofunctor (29) mentioned in \$III-D is simply defined as the composite:

$$
!=\text { Mult } \circ \text { Lin }: \operatorname{HoGames}(\star) \longrightarrow \operatorname{HoGames}(\star)
$$

on the homotopy bicategory HoGames( $(t)$ associated to the bicategory $\operatorname{Games}(\mathbb{F}, t)$.

\section{A model of differential linear logic}

In order to establish that HoGames( $(\star)$ defines a model of differential linear logic, we adapt to bicategories the description by Fiore [11] of a categorical semantics of DiLL, see also [5], [9]. First, one observes that the bicategory HoGames ( $t$ ) has finite biproducts $(\oplus, \mathbb{D})$ since its finite sums and products coincide. The multiplication and comultiplication of $! A$ are then defined using the Seely equivalence as done in Def 3.4 of [11] while the differential operator $\partial_{A}: A \otimes ! A \rightarrow ! A$ is defined just as in the model of distributors in $\$$

We are ready now to formulate the main result of the paper, a soundness theorem for differential linear logic. In order to establish the soundness property, we suppose given a right proper Quillen model category $\mathbb{S}$ equipped with a functor Sym and a span-monoidal *-autonomous $\mathbb{S}$-category $\star$ satisfying the Properties A-F formulated in \$IV] We also suppose given an exponential modality in the sense of $\$ \overline{V-C}$ (Def. 2).

Theorem (Soundness): Every formula $A$ of propositional linear logic (LL) is interpreted as a template game $\llbracket A \rrbracket$, and every derivation tree of differential linear logic (DiLL)

$$
\begin{gathered}
\pi \\
\vdots \\
\hline A_{1}, \ldots, A_{n} \vdash B
\end{gathered}
$$

is interpreted as a strategy

$$
\llbracket \pi \rrbracket: \quad \llbracket A_{1} \rrbracket \otimes \ldots \otimes \llbracket A_{n} \rrbracket \longrightarrow \quad \mid \square B \rrbracket
$$

in the bicategory HoGames( $\star \star x)$ of template games and strategies modulo homotopy. Moreover, two derivation trees $\pi$ and $\pi^{\prime}$ of DiLL related by a cut-elimination step $\pi \longrightarrow \pi^{\prime}$ define isomorphic interpretations $\llbracket \pi \rrbracket \cong \llbracket \pi^{\prime} \rrbracket$ in the bicategory HoGames $(t)$.

\section{ILLUSTRATION: ALTERNATING GAMES}

We recall in the Appendix the definition of the internal category $t_{\text {alt }}$ for alternating games and strategies, defined for $\mathbb{S}=$ Cat. The category $t_{\text {alt }}[0]$ is the category with two objects freely generated by the graph

$$
\langle\ominus\rangle \stackrel{O}{\stackrel{\leftrightarrows}{\leftrightarrows}}\langle\oplus\rangle
$$

and an alternating game $\left(A, \lambda_{A}\right)$ is thus defined as a category $A$ equipped with a functor $\lambda_{A}: A \rightarrow t_{\text {alt }}[0]$. The purpose of the objects $\langle\oplus\rangle$ and $\langle\ominus\rangle$ is thus to provide a positive or negative polarity to every object (or position) of the alternating game $A$, while the edge $O$ and $P$ are here to indicate the polarity of the trajectories and moves in the game. The free symmetric monoidal category Sym $t_{a l t}[0]$ is the category with objects of the form $w=\epsilon_{1} \cdots \epsilon_{n}$ where $\epsilon_{i} \in\{\langle\oplus\rangle,\langle\ominus\rangle\}$ for $1 \leq i \leq n$. The category $t_{\text {alt }}^{!}[0]$ is simply defined as the full subcategory of Sym $t_{\text {alt }}[0]$ consisting of the objects $w$ containing at most one negative polarity $\langle\ominus\rangle$. The functor

$$
\text { pick }[0] \quad: \quad t_{\text {alt }}^{!}[0] \longrightarrow \text { Sym } t_{\text {alt }}[0]
$$

is defined as the inclusion functor while the functor

$$
\text { pince }[0]: \quad \star_{\text {alt }}^{!}[0] \longrightarrow \star_{\text {alt }}[0]
$$

transports every word $w=\epsilon_{1} \cdots \epsilon_{n}$ to the polarity $\langle\oplus\rangle$ when $w$ contains only positive polarities $\langle\oplus\rangle$ and to the polarity $\langle\ominus\rangle$ when $w$ contains one (and thus exactly one) negative polarity $\langle\ominus\rangle$. The categories $t_{\text {alt }}^{!}[1]$ and functors pick[1] and pince[1] are defined in a similar way. One checks that the resulting structure defines an exponential modality in the sense of $\$ \sqrt{\mathrm{V}}$ for the canonical model structure on $\mathbb{S}=$ Cat. Note that the functor pick is designed to ensure the usual sequentiality requirement that at most one copy of the alternating game $A$ is of negative polarity $\langle\ominus\rangle$ in each position $a_{1} \cdots a_{n}$ of the alternating game $!_{[0]} A$.

\section{Conclusion}

We have constructed the first game model of differential linear logic (DiLL) by defining an exponential modality for the synchronization template $t=t_{\text {alt }}$ of alternating games and strategies. The construction is guided by a careful comparison with the model of generalised species, or distributors. It should be noted that a very similar (and even simpler) definition of the exponential modality $A \mapsto ! A$ for the template $\star_{\text {conc }}$ of concurrent games produces a concurrent game model of DiLL with synchronous copycat strategies, and similarly for the template $t_{\text {span }}$ of functorial spans. The construction of the model of DiLL reveals moreover a deep and unexpected connection between linear logic and homotopy theory, whose combinatorics will be explored in future work. 


\section{ACKNOWLEDGMENTS}

The author would like to thank Thomas Ehrhard, Tom Hirschowitz and Thomas Streicher for pleasant and enlightening discussions on this work.

\section{REFERENCES}

[1] Samson Abramsky, Radha Jagadeesan and Pasquale Malacaria. Full Abstraction for PCF. In Information and Computation. Vol. 163. 2000.

[2] Patrick Baillot, Vincent Danos, Thomas Ehrhard, Laurent Regnier. Believe it or not, AJM's Games Model is a Model of Classical Linear Logic. Proceedings of the 12th IEEE Symposium Logic in Computer Science (LICS), 1997.

[3] Jean Bénabou. Distributors at work. Lecture notes written by Thomas Streicher and available on his webpage, 2000.

[4] Simon Castellan, Pierre Clairambault and Glynn Winskel. Symmetry in Concurrent Games. Proceedings of the 25th EACSL Conference on Computer Science Logic and 29th ACM/IEEE Symposium Logic in Computer Science (CSL-LICS), 2014.

[5] Rick Blute, Robin Cockett, and R. A. G. Seely Differential categories. Mathematical Structures in Computer Science, 16(6):10491083,2006

[6] Clovis Eberhart and Tom Hirschowitz. What's in a game? A theory of game models. Proceedings of the 33rd Annual ACM/IEEE Symposium on Logic in Computer Science, LICS 2018.

[7] Jeffrey Egger. Quillen model structures, *-autonomous categories and adherence spaces. University of Ottawa, 2006.

[8] Thomas Ehrhard and Laurent Regnier. The differential lambdacalculus. Theor. Comput. Sci. 309(1-3): 1-41 (2003)

[9] Thomas Ehrhard. An introduction to differential linear logic: proof-nets, models and antiderivatives. Mathematical Structures in Computer Science 28(7): 995-1060 (2018)

[10] Marcelo P. Fiore, Nicola Gambino, Martin Hyland and Glynn Winskel. The Cartesian closed bicategory of generalised species of structures. Journal of the London Mathematical Society 77(1):203-220, 2007.

[11] Marcelo P. Fiore. Differential Structure in Models of Multiplicative Biadditive Intuitionistic Linear Logic. Lecture Notes in Computer Science 4583, Springer, 2007.

[12] Russell Harmer, Martin Hyland and Paul-André Melliès. Categorical Combinatorics for Innocent Strategies. Proceedings of the 22nd IEEE Symposium on Logic in Computer Science (LICS), 2007.

[13] Russell Harmer, Guy McCusker. A fully abstract game semantics for finite nondeterminism, in: Proceedings, Fourteenth Annual IEEE Symposium on Logic in Computer Science, IEEE Computer Society Press, 1999, pp. 422430.

[14] André Hirschowitz, Michel Hirschowitz and Tom Hirschowitz. A Theory for Game Theories. Proceedings of the 27th IARCS Annual Conference on Foundations of Software Technology and Theoretical Computer Science (FSTTCS), LNCS 4855, Springer Verlag, 2007.

[15] Mark Hovey. Model categories. Mathematical Surveys and Monographs, vol.63, American Mathematical Society, Providence, RI, 1999.

[16] Jim Laird, Giulio Manzonetto, Guy McCusker. Constructing differential categories and deconstructing categories of games. Inf. Comput. 222: 247-264 (2013)

[17] Damiano Mazza. Infinitary Affine Proofs. Mathematical Structures in Computer Science, 27(5):581-602, 2017.

[18] Paul-André Melliès, Asynchronous games 2: the true concurrency of innocence. Theoretical Computer Science, Special Issue Selected papers of CONCUR 2004, Volume 358, Issues 2-3, pages 200-228, 2006.

[19] Paul-André Melliès, Categorical semantics of linear logic. Panoramas et Synthèses, Tome 27, Société Mathématique de France, 2009.

[20] Paul-André Melliès. Categorical combinatorics of scheduling and synchronization in game semantics. Proceedings of the ACM on Programming Languages Issue POPL, January 2019.
[21] Paul-André Melliès, Nicolas Tabareau, Christine Tasson. An explicit formula for the free exponential modality of linear logic. Mathematical Structures in Computer Science 28(7): 1253-1286 (2018) 


\section{APPENDIX A}

THE TEMPlATE OF ALTERNATING GAMES

We describe the synchronization template $t_{\text {alt }}$ for alternating games and strategies. The category called the template of games

$$
t_{\text {game }}=t_{\text {alt }}[0]
$$

is defined as the category with two objects $\langle\oplus\rangle$ and $\langle\ominus\rangle$ freely generated by the oriented graph

$$
\langle\ominus\rangle \underset{P}{\stackrel{O}{\leftrightarrows}}\langle\oplus\rangle
$$

The category called the template of strategies

$$
t_{\text {strat }}=\star_{\text {alt }}[1]
$$

is defined as the category freely generated by the graph

$$
\langle\ominus, \ominus\rangle \underset{O_{s}}{\stackrel{P_{s}}{\leftrightarrows}}\langle\oplus, \ominus\rangle \stackrel{O_{t}}{\stackrel{P_{t}}{\leftrightarrows}}\langle\oplus, \oplus\rangle
$$

Each of the four labels $O_{s}, P_{s}, O_{t}$ and $P_{t}$ is here to describe a specific kind of Opponent and Player move:

$O_{s}$ : Opponent move played in the source game

$P_{s}$ : Player move played in the source game

$O_{t}$ : Opponent move played in the target game

$P_{t} \quad$ : Player move played in the target game

The four edges $P_{s}, O_{s}, P_{t}$ and $O_{t}$ of the graph (48) may be depicted as follows:
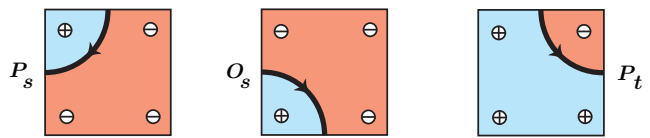

The morphisms of the category $t_{\text {strat }}$ are called scheduling trajectories. Typically, the scheduling trajectory

$$
\langle\oplus, \oplus\rangle \stackrel{O_{t}}{\longrightarrow}\langle\oplus, \ominus\rangle \stackrel{P_{s}}{\longrightarrow}\langle\ominus, \ominus\rangle \stackrel{O_{s}}{\longrightarrow}\langle\oplus, \ominus\rangle \stackrel{P_{t}}{\longrightarrow}\langle\oplus, \oplus\rangle
$$

is depicted as follows in this graphical notation:

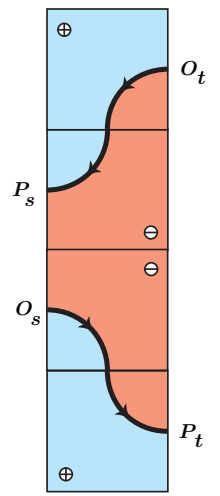

The category $t_{\text {strat }}$ comes equipped with a span of functors

$$
t_{\text {game }} \longleftarrow t_{\text {strat }} \longrightarrow t \rightarrow t_{\text {game }}
$$

where the functor $s$ is defined as the "projection" on the first component:

$$
\begin{array}{rlcc}
\langle\ominus, \ominus\rangle & \mapsto\langle\ominus\rangle & O_{s} \mapsto P & P_{s} \mapsto O \\
\langle\oplus, \ominus\rangle,\langle\oplus, \oplus\rangle & \mapsto\langle\oplus\rangle & O_{t}, P_{t} \mapsto \operatorname{id}_{\langle\oplus\rangle}
\end{array}
$$

and the functor $t$ is defined as the "projection" on the second component:

$$
\begin{array}{rlrl}
\langle\oplus, \oplus\rangle & \mapsto\langle\oplus\rangle & O_{t} \mapsto P & P_{t} \mapsto O \\
\langle\ominus, \ominus\rangle,\langle\oplus, \ominus\rangle & \mapsto\langle\ominus\rangle & O_{s}, P_{s} \mapsto \operatorname{id}_{\langle\ominus\rangle}
\end{array}
$$

The source and target functors may be illustrated as follows in our graphical notation:

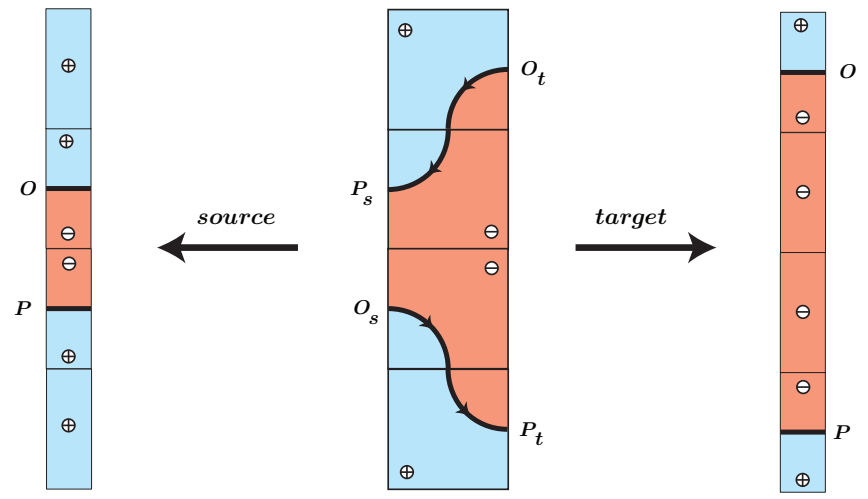

The category $\star_{\mathrm{int}}$ called the template of interactions

$$
t_{\text {int }}=t_{\text {alt }}[2]
$$

is defined by the pullback diagram below:

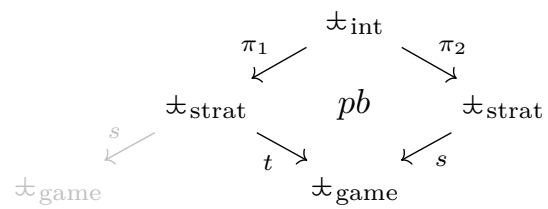

An easy computation shows that the category $t_{\text {int }}$ has four objects

$$
\langle\ominus, \ominus, \ominus\rangle \quad\langle\oplus, \ominus, \ominus\rangle \quad\langle\oplus, \oplus, \ominus\rangle \quad\langle\oplus, \oplus, \oplus\rangle
$$

and is freely generated by the following graph:

$$
\langle\ominus, \ominus, \ominus\rangle \underset{O_{s}}{\stackrel{P_{s}}{\leftrightarrows}}\langle\oplus, \ominus, \ominus\rangle \underset{P \mid O}{\stackrel{O}{\leftrightarrows}}\langle\oplus, \oplus, \ominus\rangle \underset{P_{t}}{\stackrel{O_{t}}{\leftrightarrows}}\langle\oplus, \oplus, \oplus\rangle
$$

The six edges of the graph may be depicted as follows in our graphical language:
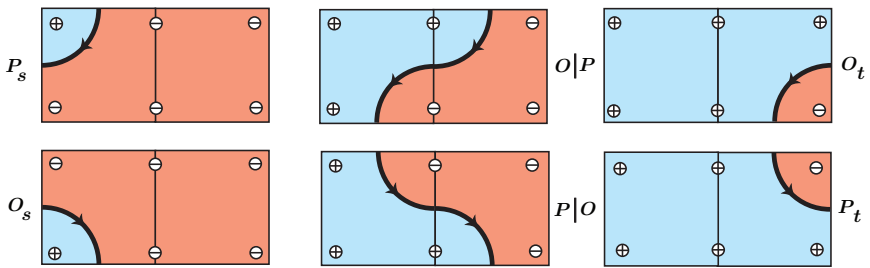

so that a typical trajectory of interactions between two alternating strategies is represented as follows: 


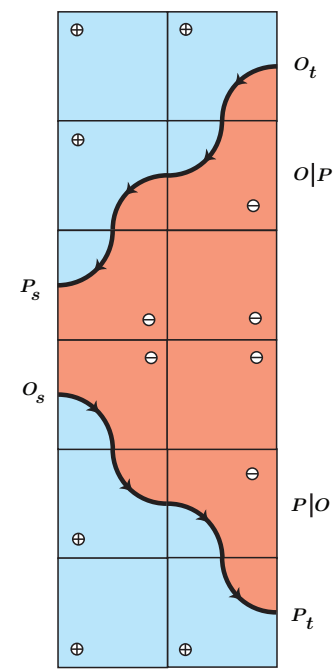

The category $t_{\text {int }}$ of interactions comes equipped with a functor

$$
m=\text { hide }: \star_{\text {int }} \longrightarrow \star_{\text {strat }}
$$

which makes the diagram below commute:

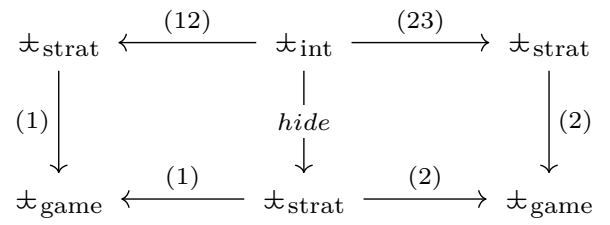

and thus defines a map of functorial spans. The functor hide is defined as the "projection" on the first and third components, and is noted (13) for that reason:

$$
\begin{aligned}
&\langle\ominus, \ominus, \ominus\rangle \mapsto\langle\ominus, \ominus\rangle \\
&\langle\oplus, \ominus, \ominus\rangle,\langle\oplus, \oplus, \ominus\rangle \mapsto\langle\oplus, \ominus\rangle \\
&\langle\oplus, \oplus, \oplus\rangle \mapsto\langle\oplus, \oplus\rangle \\
& O_{s} \mapsto O_{s} \quad P_{s} \mapsto P_{s} \\
& O|P, P| O \mapsto \operatorname{id}_{\langle\oplus, \ominus\rangle} \\
& O_{s} \mapsto O_{s} \quad P_{s} \mapsto P_{s}
\end{aligned}
$$

The action of the functor hide on the interaction trajectory above is represented as follows in our graphical language:

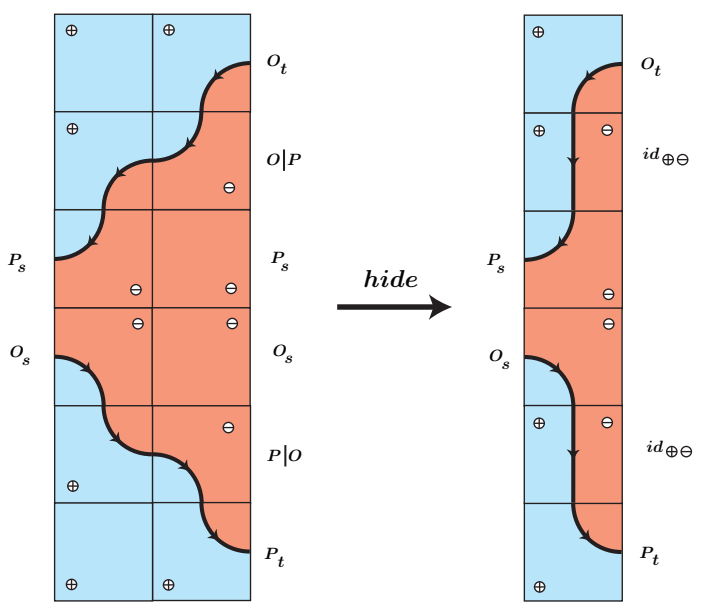

There also exists a functor

$$
e=\text { copycat }: t_{\text {game }} \longrightarrow t_{\text {strat }}
$$

which makes the diagram below commute:

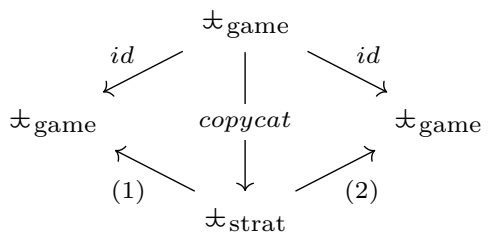

The functor copycat is defined as follows on the objects and morphisms of the category $t_{\text {game }}$ :

$$
\begin{aligned}
\langle\ominus\rangle & \mapsto\langle\ominus, \ominus\rangle & O \mapsto O_{t} \cdot P_{s} \\
\langle\oplus\rangle & \mapsto\langle\oplus, \oplus\rangle & P \mapsto O_{s} \cdot P_{t}
\end{aligned}
$$

The pair of categories

$$
t_{\text {game }}=t_{\text {alt }}[0] \quad t_{\text {strat }}=t_{\text {alt }}[1]
$$

together with the functors $s, t, m, e$ defines an internal category $t_{\text {alt }}$ in $\mathbb{S}=$ Cat. 


\section{APPENDIX B}

\section{HOMOTOPY PULLBACKS AND DIFFERENTIAL LINEAR}

LOGIC

Here, we illustrate the fact that shifting from pullbacks to homotopy pullbacks is necessary in order to interpret differential linear logic in our template game model, and more specifically, to ensure that every exponential object !A comes equipped with the expected structure of a bimonoid (= bialgebra). As we did in \$III-B, we suppose here that $\mathbb{S}=$ Cat and that $t=t_{\text {span }}$. Imagine that one decides to compute directly in the bicategory $\operatorname{Games}(\star)=\operatorname{Span}($ Cat $)$ the composite

$\operatorname{Sym} A \otimes \operatorname{Sym} A \longrightarrow \operatorname{Sym} A \longrightarrow \operatorname{Sym} A \otimes \operatorname{Sym} A$

of the two spans defining comultiplication $d_{A}$ and multiplication $m_{A}$ and formulated in (24) and (25). To that purpose, one starts by computing in $\mathbb{S}=$ Cat the pullback of the diagram

$\operatorname{Sym} A \times \operatorname{Sym} A \stackrel{\otimes_{A}}{\longrightarrow} \operatorname{Sym} A \stackrel{\otimes_{A}}{\longleftarrow} \operatorname{Sym} A \times \operatorname{Sym} A$

Because of the sequential nature of words and concatenation, the result consists of three components instead of four as one would expect of a bimonoid (or bialgebra) $! A$ in a model of differential linear logic. In order to recover the appropriate form of composition, one needs to compute instead the homotopy pullback (or in that specific case, isopullback) of the diagram. One simple way to proceed is to observe that the functor

$$
\operatorname{Sym} \nabla_{A} \quad: \quad \operatorname{Sym}(A+A) \longrightarrow \operatorname{Sym} A
$$

is an isofibration which happens to define the fibrant replacement of the functor $\otimes_{A}$ defined in (2). Then, the homotopy pullback may be computed as the (usual) pullback

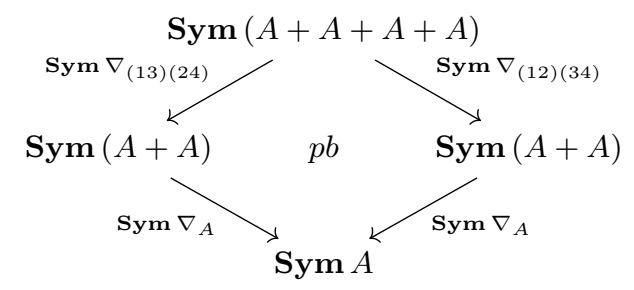

obtained as the image by the cartesian 2-monad Sym of the pullback diagram computed in Cat. This shows that the construction of a model of differential linear logic based on Span(Cat) and more generally Games( $(t)$ requires to introduce ideas coming from homotopy theory, and to replace the usual pullbacks in $\mathbb{S}$ by homotopy pullbacks in $(\mathbb{S}, \mathscr{W}, \mathscr{C}, \mathscr{F})$. 